\title{
Characteristics of a RF-Driven Ion Source for a Neutron Generator Used For Associated Particle Imaging
}

\author{
Ying $\mathrm{Wu}^{1,2}$, Paul Hurley ${ }^{3}$, Qing $\mathrm{Ji}^{1}$, Joe Kwan ${ }^{1}$, Ka-Ngo Leung ${ }^{1,2}$ \\ ${ }^{1}$ Lawrence Berkeley National Laboratory, 1 Cyclotron Road, Berkeley, CA 94720 \\ ${ }^{2}$ Department of Nuclear Engineering, University of California-Berkeley, Berkeley, CA 94720 \\ ${ }^{3}$ Special Technologies Laboratory, Santa Barbara, CA 93111
}

\begin{abstract}
We present recent work on a prototype compact neutron generator for associated particle imaging (API). API uses alpha particles that are produced simultaneously with neutrons in the deuterium-tritium $\left({ }^{2} \mathrm{D}\left({ }^{3} \mathrm{~T}, \mathrm{n}\right)^{4} \alpha\right)$ fusion reaction to determine the direction of the neutrons upon exiting the reaction. This method determines the spatial position of each neutron interaction and requires the neutrons to be generated from a small spot in order to achieve high spatial resolution. The ion source for API is designed to produce a focused ion beam with a beam spot diameter of 1-mm or less on the target. We use an axial type neutron generator with a predicted neutron yield of $10^{8} \mathrm{n} / \mathrm{s}$ for a $50 \mu \mathrm{A} \mathrm{D} / \mathrm{T}$ ion beam current accelerated to $80 \mathrm{kV}$. The generator utilizes a RF planar spiral antenna at $13.56 \mathrm{MHz}$ to create a highly efficient inductively-coupled plasma at the ion source. Experimental results show that beams with an atomic ion fraction of over $80 \%$ can be obtained while utilizing only 100 watts of RF power in the ion source. A single acceleration gap with a secondary electron suppression electrode is used in the tube. Experimental results, such as the current density, atomic ion fraction, electron temperature, and electron density, from ion source testing will be discussed.
\end{abstract}

Keywords: Neutron generator, RF plasma ion source, Associated particle imaging

PACS: 29.25.Dz, 52.77.-j, 52.50.Dg, 52.50.Qt, 52.59.Ye, 29.25.Lg

\section{INTRODUCTION}

Neutron generators that utilize rf-driven plasma ion sources have been developed at the Lawrence Berkeley National Laboratory (LBNL) [1-6]. These neutron generators are useful in the interrogation of cargo or luggage containers, which may contain explosives, contraband, or special nuclear materials. Here, the neutrons are typically produced by the deuterium-deuterium (Eqn. 1) or the deuterium-tritium (Eqn. 2) fusion reactions with a fairly large ion source that produces up to tens of $\mathrm{mA}$ of $\mathrm{D} / \mathrm{T}$ ion current. The ions are accelerated to energies of $100 \mathrm{keV}$ or higher towards a metal hydride target which can produce neutron yields of over $10^{9} \mathrm{n} / \mathrm{s}$ for $\mathrm{D}-\mathrm{D}$ and $10^{11} \mathrm{n} / \mathrm{s}$ for D-T.

$$
\begin{aligned}
& { }^{2} \mathrm{D}+{ }^{2} \mathrm{D} \rightarrow{ }^{1} n+{ }^{3} \mathrm{He}\left(E_{n}=2.45 \mathrm{MeV}, E_{3_{3 \mathrm{He}}}=0.82 \mathrm{MeV}\right) \\
& { }^{2} \mathrm{D}+{ }^{3} \mathrm{~T} \rightarrow{ }^{4} \mathrm{He}+{ }^{1} n,\left(E_{n}=14.1 \mathrm{MeV}, E_{{ }^{{ }_{H}} \mathrm{He}}=3.5 \mathrm{MeV}\right)
\end{aligned}
$$

These neutron generators require large power supplies as well as active water-cooling that makes transportation difficult. Therefore, the development of a compact neutron generator with a moderate yield that is easily portable would provide greater flexibility in the detection of contraband materials. Associated Particle Imaging (API) is a special type of active neutron interrogation that uses the deuterium-tritium neutron production reaction and takes advantage of the alpha particle that is produced in the reaction to track the neutron trajectory [7]. Since the alpha particle travels in the opposite direction of the neutron,due to conservation of linear momentum, the use of a position sensitive alpha detector can provide the neutron direction and D-T reaction time. This limits the interrogation neutrons to those that are tagged by the alpha detector traveling in the opposite direction of the alpha particles and acts as a virtual collimation for the isotropically produced neutrons [8]. Once the neutron reaches the target material, it can be inelastically scattered and produces characteristic gamma rays, which are then detected. The time between the detection of an alpha particle and the detection of a gamma ray provides the neutron flight time which can be used to locate the neutron interaction. Using this method, the location of the target material and its identity can be determined [9]. Currently, commercial API neutron generators utilize Penning ion sources that are compact and low on power consumption, but 
have a low efficiency because the ions produced are mostly molecular [10]. For these Penning ion sources, the life-time of the extraction aperture is also an issue. These sources have high anode voltages which accelerate the ions towards the cathode and cause sputtering of the extraction aperture and produce an increasingly larger beam spot as time goes on. Having a small ion beam diameter is an important parameter in API because it determines the $x-y$ resolution of the resultant image. Current API neutron generators can only achieve an initial beam diameter of about 2-mm, that will continue to increase as the operation of the source progresses. For neutron generators where the beam spot size does not matter, a low atomic ion fraction in the ion source can be overcome by increasing the ion current and beam energy. However, since API requires a small beam spot, increasing the beam current and beam energy will result in an increase in the power density on the target surface. The current API neutron generator using the Penning source has a power of nearly $6 \mathrm{~W}$, which would result in a power density $700 \mathrm{~W} / \mathrm{cm}^{2}$ for a $1-\mathrm{mm}$ beam spot, which is too high to be cooled effectively by air, and the neutron yield would decrease because the implanted deuterium/tritium ions would diffuse out of the target surface. Thus, the only way to increase the neutron yield while decreasing the beam spot size simultaneously is to increase the atomic ion fraction, and an increase from $10 \%$ to $90 \%$ of atomic ions while keeping the beam current and energy constant would increase the neutron yield by a factor of 4 [11] with the operating conditions listed for API in Table 1. The API neutron generator currently being developed will utilize a RF-driven ion source which can provide over $90 \%$ atomic ions with a $1-\mathrm{mm}$ beam spot diameter with a power density of $200-400 \mathrm{~W} / \mathrm{cm}^{2}$, which can be cooled by air. Table 1 shows the operating parameters of the RF-driven neutron generator and the operating parameters of the commercially available API neutron generator. The RF-driven neutron generator is being developed specifically to increase the atomic ion species so that the neutron yield can be made larger and the beam spot made smaller without over heating the target while also keeping the power consumption and gas pressure low in the ion source.

\begin{tabular}{lcc}
\multicolumn{3}{c}{ TABLE 1. Comparison of RF and Penning ion sources } \\
\hline \multicolumn{1}{c}{ Ion Source: } & RF & Penning \\
\hline Power consumption: & $100-125 \mathrm{~W}$ & $50 \mathrm{~W}$ \\
Ion beam current: & $20-50 \mu \mathrm{A}$ & $60 \mu \mathrm{A}$ \\
Ion beam energy: & $80 \mathrm{kV}$ & $95 \mathrm{kV}$ \\
Beam spot diameter: & $1-\mathrm{mm}$ & $2-3 \mathrm{~mm}$ \\
Atomic ion fraction: & $>80 \%$ & $<10 \%$ \\
Neutron yield: & $10^{8} \mathrm{n} / \mathrm{s}$ & $10^{7} \mathrm{n} / \mathrm{s}$ \\
Power at target: & $1.6-4 \mathrm{~W}$ & $5.7 \mathrm{~W}$ \\
\hline
\end{tabular}

\section{EXPERIMENTAL SET-UP}

A prototype of the API neutron generator schematic can be seen in Fig. 1. The RF-driven ion source is mounted on the left and the beam is extracted and accelerated towards the target on the right side. The alpha and neutron trajectories are drawn on the schematic to illustrate that this technique uses the alpha detector to tag the neutrons produced that are going in the opposite direction of the detector.

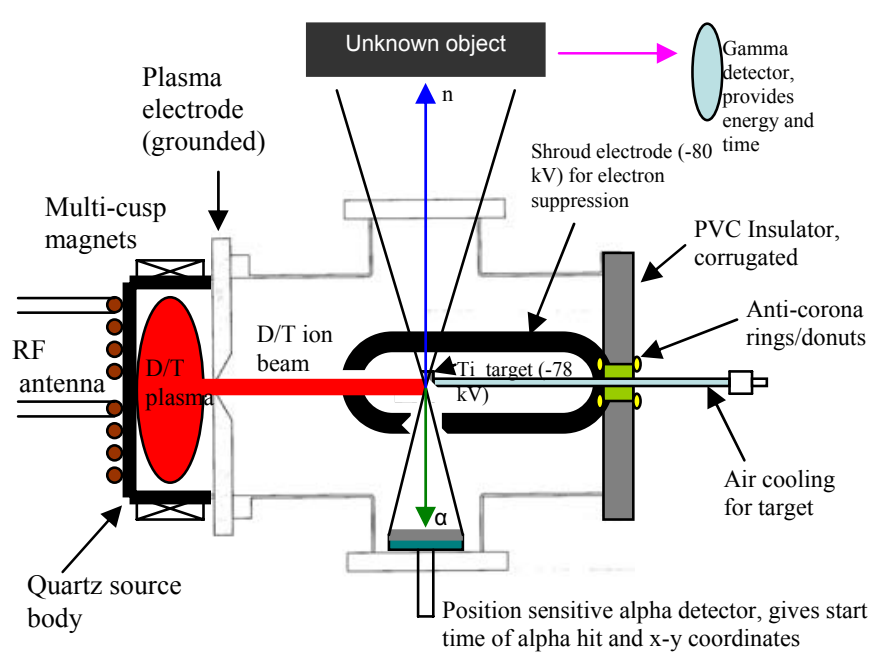

FIGURE 1. The prototype API neutron generator schematic is shown along with the trajectories of the neutrons and alphas to be used in inspection. The rf-driven ion source is located on the left and the beam is extracted and accelerated towards the target, which is negatively biased.

The tagged neutrons typically make up about 4 to $10 \%$ of the total neutrons produced. The power consumption, operating and plasma ignition pressure, atomic ion specie concentration, source cooling, size, and source durability are all factors which must be taken into consideration when constructing the ion source. In commercial compact neutron generators, the ion source used is a Penning ion source which operates at low pressures and powers, but has very low atomic ion species $(<10 \%)$ [10]. The RF ion source has been used extensively in high power, high yield neutron generator situations with very high atomic ion fraction and current density, but requires larger power supplies and extensive water-cooling on the source and target end. The rf ion source used in this API neutron generator is an improved compact RF-driven ion source which can produce high atomic fraction at low power consumption. It is $11 \mathrm{~cm}$ in diameter and 3.2 $\mathrm{cm}$ in length with 20 surrounding permanent magnet columns. The source chamber is made of quartz with an aluminum front plasma electrode. The RF power is 
delivered through a spiral, external planar antenna on the back of the source.

As with all RF-driven sources, the plasma can either be inductively or capacitively coupled, depending on the power, pressure, frequency, and the source material and geometry. Previous studies at LBNL [13], [14] have shown that sources that use internal RF antennas and solenoidal external antennas are more susceptible to capacitively coupling to the metal walls and the antenna itself, resulting in a weaker, less dense plasma. However, in the planar antenna ion source configuration, the capacitive coupling is limited between the antenna and the plasma, making it much easier to operate the source in the inductively coupled mode. Inductively coupled plasma sources are preferred for the neutron generator due to their high power efficiency and high plasma density - the same reasons that make them widely used in the semiconductor fabrication industry. In this experiment, RF frequencies of 13.56 and $27.12 \mathrm{MHz}$ were used to generate the plasma, and both hydrogen and deuterium have been used as the discharge gases. The ion species, current density, electron temperature, and electron density have all been measured for RF input power ranging from 80 to 300 watts and source pressures between 5 to $20 \mathrm{mT}$.

\section{RESULTS AND DISCUSSION}

Table 2 shows the ignition and operating pressures of deuterium at both 13.56 and $27.12 \mathrm{MHz}$ for a fixed RF power of 100 watts.
TABLE 2. Comparison of 13.56 and $27.12 \mathrm{MHz}$ RF

\begin{tabular}{lcc}
\multicolumn{1}{c}{ Frequency: } & $\mathbf{1 3 . 5 6} \mathbf{~ M H z}$ & $\mathbf{2 7 . 1 2} \mathbf{~ M H z}$ \\
\hline Ignition Pressure (for $\mathrm{D}_{2}$ ) & $30 \mathrm{mT}$ & $8 \mathrm{mT}^{*}$ \\
Minimum Pressure $\left(\right.$ for $\mathrm{D}_{2}$ ) & $8.5 \mathrm{mT}$ & $5.1 \mathrm{mT}^{*}$ \\
Ignition Pressure (for $\mathrm{H}_{2}$ ) & $50 \mathrm{mT}$ & $10 \mathrm{mT}^{*}$ \\
Minimum Pressure (for $\mathrm{H}_{2}$ ) & $12.5 \mathrm{mT}$ & $7 \mathrm{mT}^{*}$ \\
\hline
\end{tabular}

*Denotes plasma was capacitively coupled

Both the ignition and operating pressures are lower for the $27.12 \mathrm{MHz}$ than for the $13.56 \mathrm{MHz}$ plasma, but the plasma starts in capacitively coupled mode, and does not transition into inductively coupled mode until the power is increased to 300 watts. The RF input power can be reduced after the plasma changes to inductively coupled mode. The ignition and operating pressures are lower for deuterium than for hydrogen, due to the fact that the ion confinement time is longer as a result of the larger deuterium mass.

The electron density and electron temperature have been measured with an RF-compensated Langmuir probe as a function of power for deuterium and hydrogen at $13.56 \mathrm{MHz}$, as shown in Fig. 2a and 2b. The electron density increases with power and pressure, due to the increased ionization from more frequent collisions, which occur at higher power and greater number of neutrals, respectively. The electron temperature decreases with RF power and pressure due to the increase in ion and electron density because the mean free path is lowered and subsequently does not have enough time to accelerate to higher energies before colliding with another particle. For deuterium, electron and ion density are higher and the electron temperature is lower compared to hydrogen as a result of the mass difference.
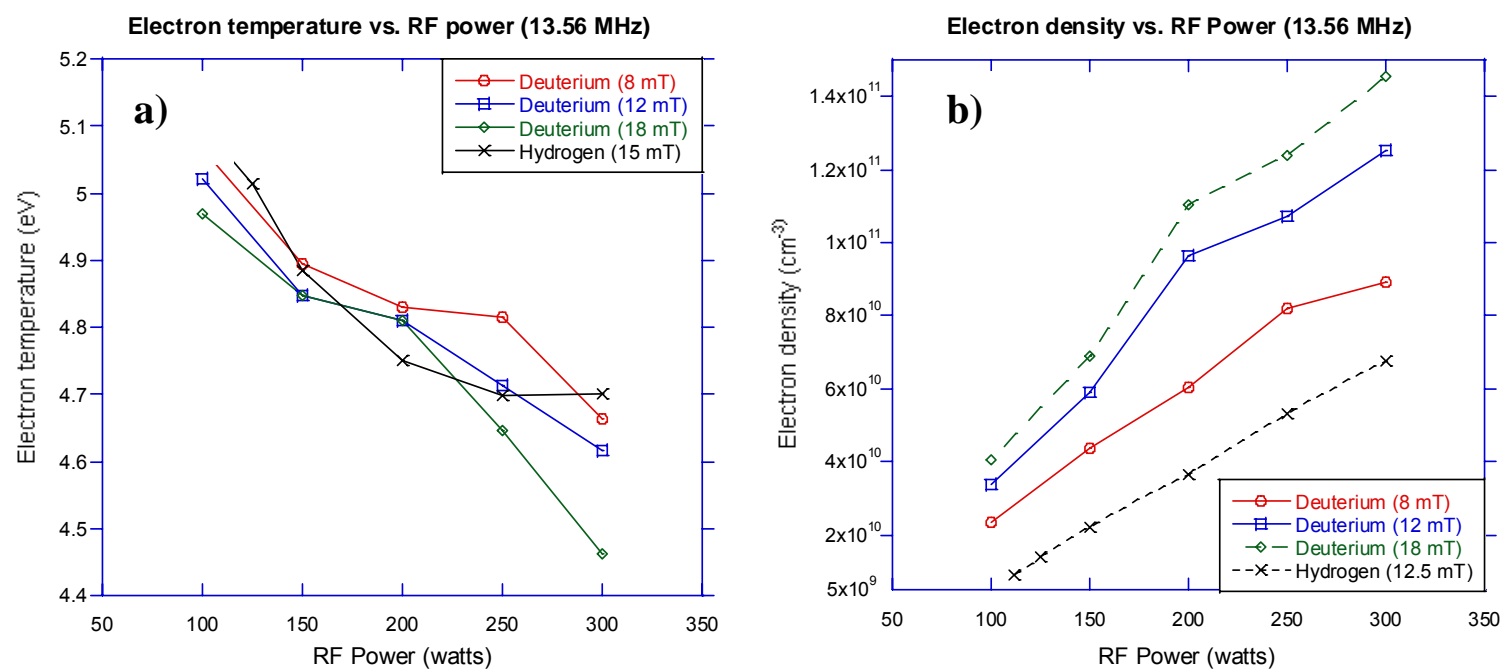

FIGURE 2. The electron temperature (a) and electron density (b) as a function of input rf power for deuterium and hydrogen It can be seen that the electron density increases with both the power and pressure. The electron density is also lower for hydrogen, which is expected since the current density is also lower. The electron temperature decreases with increasing pressure and also with increasing power. The difference in electron temperature between deuterium and hydrogen is negligible. 
The ion species of the plasma were analyzed using a mass spectrometer that filtered each species according to mass. A plot of the atomic fraction vs. power and pressure for deuterium and hydrogen is shown in Fig. 3.

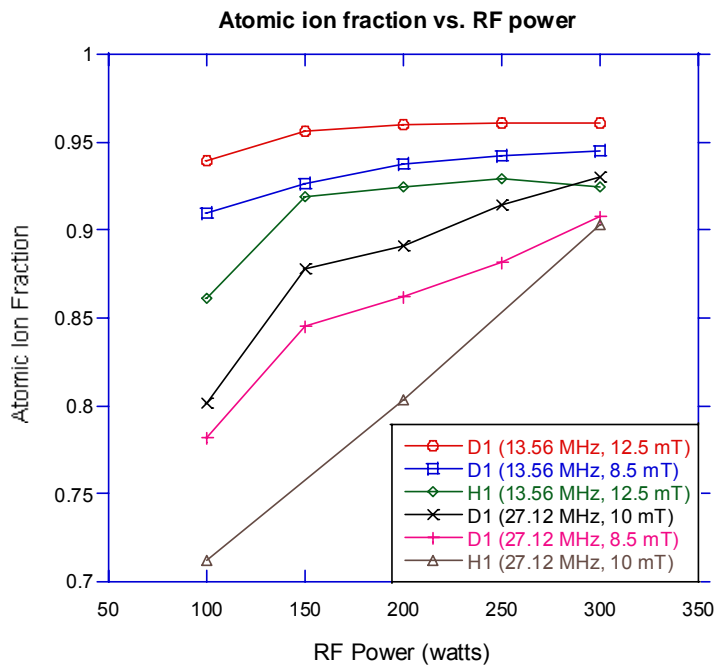

FIGURE 3. A plot of the atomic ion fraction vs. power for hydrogen and deuterium at both 13.56 and $27.12 \mathrm{MHz}$. It can be seen that the $13.56 \mathrm{MHz}$ rf produces $10-15 \%$ higher atomic fraction than $27.12 \mathrm{MHz}$ at low powers, but at higher powers the difference becomes roughly $5 \%$. The difference in atomic fraction between hydrogen and deuterium also decreases from about $7 \%$ at 100 watts to $3 \%$ at 300 watts.

From this, we can see that the atomic fraction increases with increasing plasma density and decreases with electron temperature. It may seem counterintuitive that a lower electron temperature would result in higher atomic ion fraction. However, looking at table 3, it can be seen that the dissociation energy of $\mathrm{H}_{2}$ is around $10 \mathrm{eV}$ and the ionization energy of $\mathrm{H}$ is $13.6 \mathrm{eV}$ while the ionization energy of $\mathrm{H}_{2}$ is $15.6 \mathrm{eV}$. This means that for partially ionized plasmas, an increase in the electron temperature would suppress the dissociation of $\mathrm{H}_{2}$ to $\mathrm{H}$ atoms and increase $\mathrm{H}_{2}{ }^{+}$ion formation. Therefore, the atomic ion fraction should increase with increasing electron density and decreasing electron temperature, which matches the global model and simulation done in [14] and [15]. The highest atomic ion fraction can be achieved using 13.56 MHz with a deuterium fill gas. The atomic ion fraction also increases with increased RF power and slightly with pressure. However, due to the design of the sealed neutron generator, the pressure needs to be minimized, but the atomic ion fraction is still sufficient at low pressures $(>85 \%)$.
TABLE 3. Hydrogen Ion Formation Reactions.

\begin{tabular}{lc}
\multicolumn{1}{c}{ Reactions } & Electron energy \\
\hline $\mathrm{H}+\mathrm{e} \rightarrow \mathrm{H}^{+}+2 \mathrm{e}$ & $13.6 \mathrm{eV}$ \\
$\mathrm{H}_{2}+\mathrm{e} \rightarrow \mathrm{H}_{2}^{+}+2 \mathrm{e}$ & $10.6 \mathrm{eV}$ \\
$\mathrm{H}_{2}+\mathrm{e} \rightarrow 2 \mathrm{H}+\mathrm{e}$ & $15.9 \mathrm{eV}$ \\
$\mathrm{H}_{2}^{+}+\mathrm{H}_{2} \rightarrow \mathrm{H}_{3}^{+}+\mathrm{H}$ & $0 \mathrm{eV}$ (no electron needed) \\
\hline
\end{tabular}

The RF frequency used also has an effect on the atomic fraction. For a RF frequency of $13.56 \mathrm{MHz}$, the current density and the atomic ion fraction increase greatly when the plasma is in the inductively coupled mode, which is easily achieved at this frequency. When the frequency was increased to $27.12 \mathrm{MHz}$, the plasma could ignite at pressures of less than $10 \mathrm{mT}$, but the plasma was capacitively coupled, and the power must be increased to 200-300 Watts before the transition to inductively coupled mode occurs and the power can then be decreased. In the inductively coupled mode, the plasma is denser, brighter, and has low sheath voltages, leading to lower electron energies in the plasma sheath. Capacitively coupled plasmas are dimmer, less dense (by one or two orders of magnitude), and have high sheath voltages which cause greater electron energies in the sheath [16]-[17]. Therefore, the source should be operated in the inductively coupled regime because the plasma density increases by an order of magnitude and the atomic ion fraction increases by over $25 \%$ compared to the capacitively coupled regime.

Since the final design of the neutron generator will employ a D-T gas mixture in the ion source, hydrogen and deuterium were tested to investigate the effect of gas mixing on atomic fraction. While it is true that a mixed $\mathrm{D} / \mathrm{T}$ beam will create some neutrons from the $\mathrm{D}-\mathrm{D}$ and T-T reactions, the cross section for D-T is over 300 times greater, and the impact of the D-D and $\mathrm{T}-\mathrm{T}$ neutrons is insignificant due to the lower cross sections and lower neutron energies. The advantages of using a mixed $\mathrm{D} / \mathrm{T}$ beam and a self-loading target is that the life-time will be longer than using a pure deuterium beam with a pre-loaded tritium target. For pure hydrogen gas, there are three possible ion species, but with a deuterium and tritium mixture, there are 9 possible species, with 7 different masses. With a hydrogen and deuterium mixture, there are 9 possible species, and only 6 possible masses. Although this poses a difficulty in determining the exact species of all ions, the mass spectrum should identify two large peaks at mass 1 and 2 and little to no peaks for masses 3 through 6. Fig. 4 shows the plot of the mass spectrum of the deuterium-hydrogen gas mixture at 100 watts and $20 \mathrm{mT}$, indicating that the plasma was dominated by masses 1 and 2, confirming that the high atomic fraction remains for a gas mixture. Since the amount of $\mathrm{D}_{2}^{+}$ions is very small, one can conclude 
that the amount of $\mathrm{H}_{2}{ }^{+}$ions is also minimal. Thus the mass 2 fully dominated by $\mathrm{D}^{+}$ions.

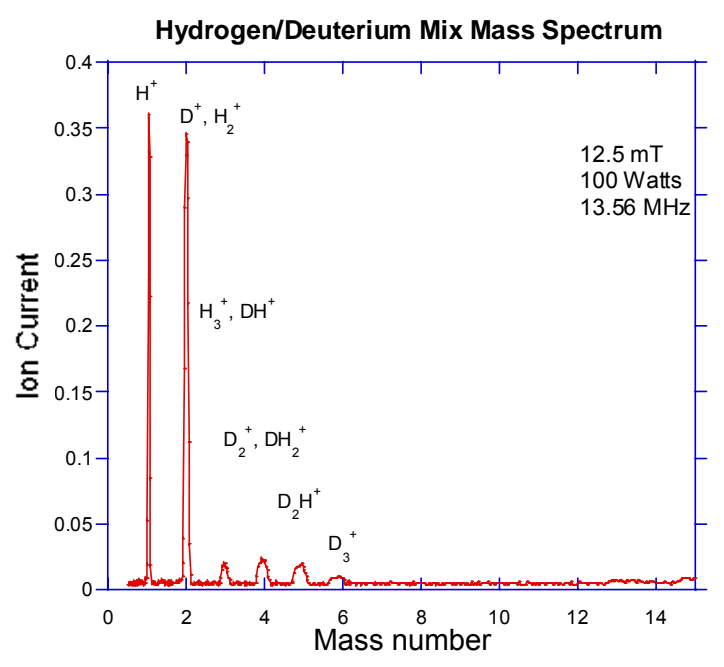

\section{ACKNOWLEDGMENTS}

The author would like to thank Steve Wilde, Paul Wong, Tom McVeigh, and Will Waldron for their technical support. This work was supported by the DOE under contract DE-AC02-05CH11231 and the DIA under contract number HHM40271611.

\section{REFERENCES}

1. J. Reijonen, Appl. Radiat. Isotopes 63, Issues 5-6, $8^{\text {th }}$ International Conference on Applications of Nuclear Techniques, 2005, pp. 757-763.

2. J. Reijonen, Nucl. Instru. and Meth. in Phys. Res. B, vol. 261, no. 1-2, Apr. 2007, pp. 272-276.

3. B. A. Ludewigt, Nucl. Instru. and Meth. in Phys. Res. B, vol. 261, no. 1-2, Apr. 2007, pp. 830-834.

4. K. N. Leung, Rev. Sci. Instrum. 71, 2000, pp. 1064.

5. J. Reijonen, K. N. Leung, G. Jones, Rev. Sci. Instrum. 73, 2002, pp. 934.

6. K. N. Leung, Rev. Sci. Instrum. 69, 1998, pp. 998.

FIGURE 4. A mass spectrum of a 50/50 hydrogen-deuterium mix 7. A. Beyerle, J.. P. Hurley, L. Tunnell, Nucl. Instr. and plasma at a pressure of $12.5 \mathrm{mT}$ and 100 watts of input $\mathrm{rf}$ power. The Meth. A, 299, 1990, pp. 458.

plasma is dominated by mass 1 and 2, which are the atomic ion spegies A. Beyerle, Nuclear Science Symposium and Medical of hydrogen and deuterium. The other 7 possible species account for approximately $10 \%$ of the total ions.

\section{SUMMARY}

A prototype compact RF-driven neutron generator for API has been built and the ion source characteristics measured. Experiments show that the RF-driven ion source using quartz as the source material and an external planar antenna will be able to provide an upgrade in source efficiency at both 13.56 and $27.12 \mathrm{MHz}$ over the Penning source by producing over $85 \%$ atomic ions and thus allowing the neutron yield to increase and the beam spot to decrease without overheating the target. Experiments with deuterium and hydrogen have shown that using deuterium at the same power and pressure as hydrogen yields a higher current density, a higher atomic ion fraction, higher electron density, and lower operating pressures. Therefore, it is expected that using a mixture gas of deuterium and tritium would result in even higher atomic ion fraction and lower operating pressures. This source also demonstrated that a mixture of deuterium and hydrogen has no effect on the ion species as the atomic ions dominated. A deuteriumtritium mixture is expected to behave similarly. Future work will involve measurement of the beam spot at 80 $\mathrm{kV}$, testing the neutron yield with deuterium, and operating the system in a sealed environment. Imaging Conference, 1991., Conference Record of the 1991 IEEE , vol., no., pp.1298-1304 vol.2, 2-9 Nov 1991.

9. L. E.Ussery and C. L.Hollas, "Design and Development of the Associated-Particle Three-Dimensional Imaging Technique", Rep. LA-12847- MS (UC-706 and UC-700), Los Alamos National Laboratory, Los Alamos, NM.

10. D. L. Chichester, Nuclear Instruments and Methods in Physics Research Section B: Beam Interactions with Materials and Atoms, 241, Issues 1-4, Dec. 2005, pp. 753-758.

11. J. M. Verbeke, Appl. Radiat. Isot. 53, 2000, pp. 801809.

12. Q. Ji, J. Vac. Sci. Technol. B 20(6), 2002, pp. 2717.

13. X. Jiang, J. Vac. Sci. Technol. B 21(6), 2003, pp. 2724.

14. C. F. Chan, C. F. Burnell, W. S. Cooper, J. Appl. Phys,. 54, 1983, pp. 6119.

15. O. Fukumasa, J. Phys. D: Appl. Phys. 18, 1985, pp. 2433.

16. U. Kortshagen, N. D. Gibson, J. E. Lawler, J. Phys. D: Appl. Phys. 29, 1996, pp. 1224-1236.

17. M. M. Turner and M. A. Lieberman, Plasma Sources Sci. Technol., vol. 8, 1999, pp. 312-324. 


\section{DISCLAIMER}

This document was prepared as an account of work sponsored by the United States Government. While this document is believed to contain correct information, neither the United States Government nor any agency thereof, nor The Regents of the University of California, nor any of their employees, makes any warranty, express or implied, or assumes any legal responsibility for the accuracy, completeness, or usefulness of any information, apparatus, product, or process disclosed, or represents that its use would not infringe privately owned rights. Reference herein to any specific commercial product, process, or service by its trade name, trademark, manufacturer, or otherwise, does not necessarily constitute or imply its endorsement, recommendation, or favoring by the United States Government or any agency thereof, or The Regents of the University of California. The views and opinions of authors expressed herein do not necessarily state or reflect those of the United States Government or any agency thereof or The Regents of the University of California. 